\title{
Tuberculosis latente
}

\author{
JUAN C. RODRÍGUEZ D.*
}

\section{Latent tuberculosis}

Tuberculosis continues being a huge public health problem. It has been estimated that worldwide every year about 9 million people adquire the disease and 2 million of deaths are because of tuberculosis. Exposure to Mycobacterium tuberculosis causes active disease in nearly $10 \%$ of the people. In the remaining $90 \%$ of the exposed population the immune response inhibits $M$. tuberculosis multiplication. In part of the exposed people ( 40\%) some bacilli are not killed remaining in a non-replicating condition, generating the so called latent tuberculosis infection. The inactive bacilli can regain their vitality (replication capacity) and cause active tuberculosis if the immune response is disrupted. Targeted screening and treatment for latent tuberculosis infection has been recognized as an effective strategy for tuberculosis control. Until recently, the only test available for the detection of latent tuberculosis infection was the tuberculin (PPD) skin test. Recently it has been developed an in vitro T-cell-based interferon-gamma release assay (IGRA). This assay use antigens specific to M. tuberculosis and is not affected by previous BCG vaccination, making it a useful screening test for latent tuberculosis in BCG-vaccinated populations. FDA has approved two commercial IGRAs: a) QuantiFERON-TB Gold (QFT, Cellestis) which simplified variation is called Quantiferon T in Tube (QFT-G-IT) and b) T-SPOT $T B$ test (Oxford, Immunotec). According to the Chilean National Program of Tuberculosis Control, therapy for latent tuberculosis infection is daily administration of isoniazid for 9 months; administered regularly this therapy gives over 90\% of protection. Another option used in USA and Europe is to prescribe rifampicin for 4 months. Identification of latent tuberculosis carriers and their treatment is a pivotal action in the efforts for decreasing tuberculosis incidence in developing countries with low or intermediate rates of tuberculosis incidence.

Key words: Latent tuberculosis/diagnosis, latent tuberculosis/treatment, molecular diagnostic techniques.

\section{Resumen}

La tuberculosis continúa siendo un problema enorme en salud pública. Se ha estimado que anualmente en todo el mundo 9 millones de personas contraen esta enfermedad y que 2 millones de muertes se deben a la tuberculosis. La exposición al M. tuberculosis causa la enfermedad en cerca del $10 \%$ de la población expuesta. En el $90 \%$ restante de los expuestos la respuesta inmune inhibe la multiplicación del M. tuberculosis. En una parte de las personas expuestas ( 40\%) algunos bacilos no son muertos permaneciendo en una condición que no les permite replicarse, generándose así la infección denominada tuberculosis latente. Los bacilos inactivos pueden recuperar su vitalidad (capacidad de replicación) y causar una tuberculosis activa, si la respuesta inmune está alterada. La detección y el tratamiento de la infección tuberculosa latente han sido reconocidos como objetivos estratégicos efectivos en el control de la tuberculosis. En el pasado reciente, el único test disponible para la detección de infección tuberculosa latente era el test cutáneo con tuberculina (PPD). Recientemente se ha desarrollado un ensayo basado en la liberación "in vitro" de interferón-gama (IGRA) por células T. Este ensayo usa antígenos específicos del M. tuberculosis y no es interferido por la vacunación previa con BCG, lo que lo convierte en un test de tamizaje útil en poblaciones vacunadas con BCG. La FDA ha aprobado dos IGRAs comerciales: a) QuantiFERON-TB Gold (QFT, Cellestis) cuya variación simplificada se denomina Quantiferon T in Tube (QFT-G-IT) y b) el T-SPOT TB test (Oxford, Immunotec). Según el Programa Nacional de Control de la Tuberculosis el tratamiento para la infección tuberculosa latente

* Servicio Médico-Quirúrgico Respiratorio. Instituto Nacional del Tórax. Santiago, Chile. 
es la administración diaria de isoniacida por 9 meses; este tratamiento proporciona una protección superior al 90\% si es administrado regularmente. Otra opción, usada en EE.UU. y Europa es prescribir rifampicina por 4 meses. La identificación y el tratamiento de los portadores de tuberculosis latente es una acción crucial en los esfuerzos para disminuir la incidencia de tuberculosis en países en desarrollo con tasas de incidencia de tuberculosis intermedias o bajas.

Palabras clave: Tuberculosis latente/diagnóstico, tuberculosis latente/tratamiento, técnicas diagnósticas moleculares.

\section{Introducción}

La tuberculosis sigue siendo, aún hoy, un enorme problema de salud pública. Anualmente en el mundo enferman más de nueve millones de personas de esta enfermedad y mueren alrededor de dos millones de ellas. Aunque ya se ha descubierto el genoma completo del Mycobacterium tuberculosis, su agente causal, no hemos logrado controlar la epidemia ${ }^{1,2}$.

Actualmente, se estima que un tercio de la humanidad está infectada con el bacilo tuberculoso y que en los países con buen control de la enfermedad, la mayoría de los casos provienen de la reactivación de una tuberculosis latente. Así, por ejemplo, se acepta que más del $80 \%$ de los casos nuevos que se diagnostican en Estados Unidos provienen de la reactivación endógena de una infección tuberculosa ${ }^{3}$. El objetivo de esta revisión es hacer una actualización sobre tuberculosis latente.

\section{¿Qué es la tuberculosis latente?}

El año 1907 Clemens Von Pirquet, un pediatra suizo, publicó en JAMA un manuscrito sobre un test de alergia para el diagnóstico de tuberculosis en niños. Observó que este examen tenía una sensibilidad de $60 \%$ en los casos de tuberculosis, pero también observó que un 35\% de niños sin manifestaciones clínicas de la enfermedad tenían el test positivo. Así, concluyó que los individuos con reacción positiva a la tuberculina, sin evidencias de enfermedad, tendrían una tuberculosis latente, es decir, mostraban una respuesta inmunológica al bacilo, sin tener la enfermedad ${ }^{4}$.

Con la aparición posterior de la vacuna BCG, la interpretación de la reacción a la tuberculina (PPD) se complicó, ya que en los sujetos vacunados el test también se hace positivo. La duración del efecto de la BCG sobre el PPD parece depender de la edad en que se vacuna. Así, si la BCG se coloca al nacer, a los 10 años sólo el $1 \%$ de los vacunados tendrá un PPD positivo atribuible a la vacuna; en cambio, si se vacuna después de los 2 años, el 20\% conservará la positividad a los 10 años. Por otra parte, cuando se vacuna más de una vez, como ocurre en algunos países en los que se vacunaba hasta tres veces, el efecto sobre el PPD es más prolongado ${ }^{4-6}$.

Así, la interpretación del test de tuberculina tiene algunos inconvenientes:

1. Su especificidad es baja en los individuos vacunados con BCG o infectados por micobacterias ambientales, ya que el PPD tiene una gran cantidad de antígenos y algunos son compartidos también por otras micobacterias no tuberculosas, en tanto que su sensibilidad disminuye en forma significativa en pacientes con algún grado de inmunodepresión ${ }^{2}$.

2. La interpretación del tamaño de la reacción es variable. Así, en inmunodeprimidos se suele considerar positiva una induración de $5 \mathrm{~mm}$ o más, siendo $10 \mathrm{~mm}$ el límite para considerarla positiva en sujetos normales. Los valores sobre $10 \mathrm{~mm}$, sólo significan mayor probabilidad de haber sido infectado por $M$. tuberculosis, pero no parecen estar asociados a un mayor riesgo de enfermar ${ }^{7}$.

3. Como es un test in vivo, es afectado por el efecto anamnéstico, de memoria o Booster. Hablamos de probable efecto Booster cuando la variación entre la primera y la segunda lectura de la reacción es mayor de $6 \mathrm{~mm}^{5}$. Este fenómeno tiene su mayor expresión cuando la prueba se repite entre una a cinco semanas después.

4. Cuando se repite el test la reacción puede variar, ya sea por disminución (negativización) o por aumento (conversión). El aumento puede deberse simplemente al azar (diferente forma de realizar el test o de leerlo), a un estímulo de recuerdo inmunológico o simplemente a una nueva infección.

Hasta hace sólo algunos años el diagnóstico de tuberculosis latente dependía exclusivamente del test de tuberculina, pero en la actualidad, se han agregado los IGRAs (Interferon gamma release assays), que son pruebas que miden la liberación de interferón al poner en contacto la sangre del individuo infectado con antígenos casi exclusivos del bacilo del $M$. tuberculosis s,8-10,26. $^{2}$.

Los IGRAs habitualmente utilizan ESAT-6 
(early secreating antigen), CFP-10 (culture filtrate protein) y algunos también TB7.7. Estos antígenos no los tiene el Mycobacterium bovis de la BCG, aunque son compartidos por algunas otras micobacterias ambientales (M. kansasii, $M$. szulgai, $M$. marinum y $M$. riyadhense).

Actualmente, existen dos IGRAs aprobados por la FDA (Foods and Drugs Administration) de Estados Unidos: QuantiFERON-TB Gold (QFT, Cellestis) o su variación más simplificada, Quantiferon T in Tube (QFTGIT), y T-SPOT TB test (Oxford, Immunotec).

El QFT mide el interferón gama en el sobrenadante de una suspensión celular, mientras el T-SPOT determina el número de células que están produciendo interferón, después de incubar la muestra con los antígenos señalados².

El uso de antígenos específicos del $M$. tuberculosis evita la interferencia de la vacuna BCG y el efecto Booster; además es un procedimiento más sencillo que el PPD y soluciona muchas de sus limitaciones técnicas y de interpretación.

No existe un Gold Standard para el diagnóstico de infección tuberculosa, ya que los bacilos latentes son imposibles de demostrar con las tecnologías disponibles hoy día, que sólo miden la respuesta inmunológica del huésped, pero los IGRAs parecen ser más sensibles que el PPD, y como son negativos en los inoculados con la vacuna BCG, obviamente son más específicos. Existe una infinidad de publicaciones sobre el rendimiento de los IGRAs en las más diversas situaciones epidemiológicas, por lo que no es fácil comparar los resultados obtenidos con las distintas técnicas. Así, las cifras encontradas en países de alta prevalencia no son extrapolables a las publicadas en aquellos que han logrado un mayor control de la enfermedad. Por otra parte, el grado de inmunosupresión de los individuos en los cuales se ha hecho el estudio, suele ser muy diferente en los distintos trabajos, lo que también puede explicar algunas de las diferencias encontradas entre el PPD y los IGRAs.

La TB NET ha publicado estudios en contactos de pacientes tuberculosos (Tabla 1$)^{10}$.
El resultado de los test IGRAs varía en el tiempo. Así personas con reacciones positivas pueden negativizarse con el tiempo, lo que ha originado un nuevo concepto, el de las infecciones transitorias. La positividad de los IGRAs puede demorar más de dos semanas en aparecer después del contacto con la fuente de contagio. También hay contactos que nunca se hacen positivos.

La TB NET también ha analizado los resultados de los IGRAs en comparación al PPD en pacientes con diferentes cuadros de inmunodepresión: HIV, enfermedad renal avanzada, artritis reumatoide, trasplantados de órganos sólidos y trasplantados de médula ósea ${ }^{11}$. En general, en inmunodeprimidos, los IGRAs son algo mejores que el PPD y el ELISPOT levemente superior al QTFGIT. Estos tests también se comportan de manera diferente en distintas enfermedades $y$, como siempre, hay un porcentaje de resultados indeterminados.

\section{¿Se puede predecir qué sujetos van a enfermar?}

Hay que reconocer que hoy día no disponemos de un test con una capacidad predictiva de enfermedad tuberculosa satisfactoria ${ }^{12,26}$. En general el valor predictivo positivo tanto del PPD como de los IGRAs, es bajo, aunque parece ser ligeramente superior con los IGRAs.

\section{¿Cómo se comportan los individuos al contacto con el bacilo?}

De acuerdo con las conocimientos actuales, se cree que en el contacto de un individuo sano con un paciente con tuberculosis activa puede ocurrir algunas de las posibilidades señaladas en la Figura $1^{8}$.

Un individuo en contacto con un enfermo tuberculoso puede ser o no ser infectado, dependiendo del grado de exposición y de los mecanismos inmunológicos innatos del huésped ${ }^{2}$. Un porcentaje importante, que se estima en alrededor

Tabla 1. PPD e IGRAs en estudios de contactos

\begin{tabular}{|lccc|}
\hline Test & Especificidad**(\%) & VPP***(\%) & VPN****(\%) \\
PPD & 88,7 & $2,3-3,3$ & 99,7 \\
QFTGIT & 99,4 & $2,8-14,3$ & 99,8 \\
ELISPOT & 98,0 & $3,3-10,0$ & 97,8 \\
\hline
\end{tabular}

**La especificidad se calcula en individuos con escaso a nulo riesgo de contacto. ***VPP. Valor predictivo positivo según número de individuos con test positivo que enferman. ****VPN. Valor predictivo negativo según número de individuos con test negativo que no enferman. 
del 60\%, a pesar de la exposición a la fuente de contagio no desarrolla nunca una respuesta inmunológica, es decir, persiste PPD negativo. La explicación de este hecho es desconocida.

Cuando ocurre la infección el sujeto puede controlarla o por el contrario la infección puede sobrepasar sus mecanismos defensivos y progresar a enfermedad. Si la controla puede eliminarla completamente sin dejar huella o quedar con algunos bacilos vivos, sin que éstos logren ser eliminados completamente, pero sin capacidad de producir enfermedad. Los portadores de esta infección controlada tienen lo que denominamos una infección latente; tienen respuesta inmunológica al bacilo tuberculoso, pero no están enfermos. De todos los individuos que son infectados sólo un 10 \% progresa a enfermedad y la mayoría lo hace en los primeros años que siguen a la infección. Un 15\% de los que enferman, es decir, un 1,5\% de los infectados hace una enfermedad grave. Si lo expresamos en números, de 100 expuestos, 40 se infectarán y se harán PPD (+), 4 de éstos enfermarán de tuberculosis y uno de cada 8 de estos enfermos desarrollará una tuberculosis grave.

\section{¿Cómo se genera la respuesta inmune?}

Después de la llegada del bacilo de Koch a los espacios alveolares, éste es captado por los macrófagos que liberan citoquinas y quemoquinas que son la señal de que se ha producido la infección. Monocitos, linfocitos y neutrófilos migran al sitio de la infección, pero al no estar activados, son incapaces de matar al microorganismo ${ }^{2}$.

El M. tuberculosis es capaz, en este estado, de bloquear la fusión de los fagosomas con los liso-

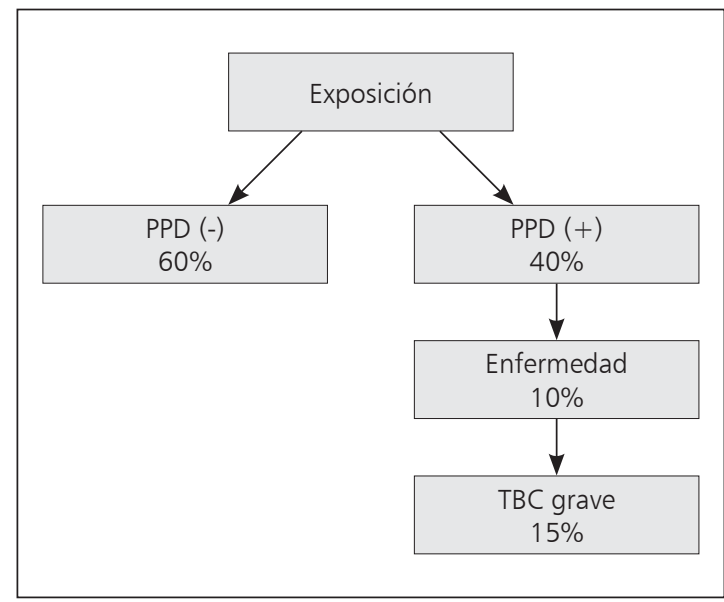

Figura 1. Evolución de los contactos de un enfermo contagioso. somas de los macrófagos, multiplicándose dentro de los fagosomas y eventualmente produciendo la necrosis del macrófago, escapando al medio extracelular. Los bacilos así liberados se multiplican extracelularmente y son fagocitados por otros macrófagos, que tampoco son capaces de controlar al bacilo ${ }^{13,14}$. Este proceso se mantiene en pacientes con inmunidad débil, hasta llegar a producir una enfermedad activa.

Sin embargo, la mayoría de las personas entre las 2 y las 8 semanas de ocurrido el contagio, desarrollan una respuesta celular inmune que es capaz de controlar la infección (Figura 2).

Los macrófagos y las células dendríticas migran a los ganglios, donde presentan los antígenos a los complejos de histocompatibilidad (MHC clase II) de los linfocitos tanto CD4 como CD8. En los ganglios los linfocitos son estimulados para diferenciarse en productores de interferón gama, T helper, Th1 o TC1 citotóxico. Al mismo tiempo, las células B se transforman en secretoras de anticuerpos específicos contra el bacilo (Figura 2).

Todas estas células llegan al torrente sanguíneo y migran posteriormente a los sitios de infección en el pulmón. En este momento la respuesta inmunológica se hace evidente, como una manifestación de hipersensibilidad retardada y el PPD y los IGRAs se hacen positivos.

La presencia de linfocitos Th 1 activados en el sitio de la infección es fundamental para la formación de los granulomas característicos de la tuberculosis. Los macrófagos activados se transforman en células epitelioides rodeadas por una corona de linfocitos. Algunas de ellas aparecen como células gigantes de Langhans².

Los granulomas son estructuras dinámicas, continuamente hay células que mueren y sus restos son removidos. Se cree que finalmente se produce un equilibrio entre las micobacterias que se multiplican y aquellas que se adaptan a las condiciones adversas dentro del macrófago, al que no destruyen completamente, pero siendo incapaces de multiplicarse. Así, los bacilos llegarían a un estado persistente de no replicación. Cuando se bloquean las moléculas de adhesión, como ocurre por ejemplo con las drogas anti FNT (factor de necrosis tumoral), el granuloma se desintegra y libera su contenido ${ }^{36}$.

\section{¿Cuál es la evidencia de que existan bacilos vivos en la tuberculosis latente?}

En primer lugar, se sabe que los individuos PPD positivo tienen un riesgo de enfermar que es entre tres a cinco veces mayor que el de un 


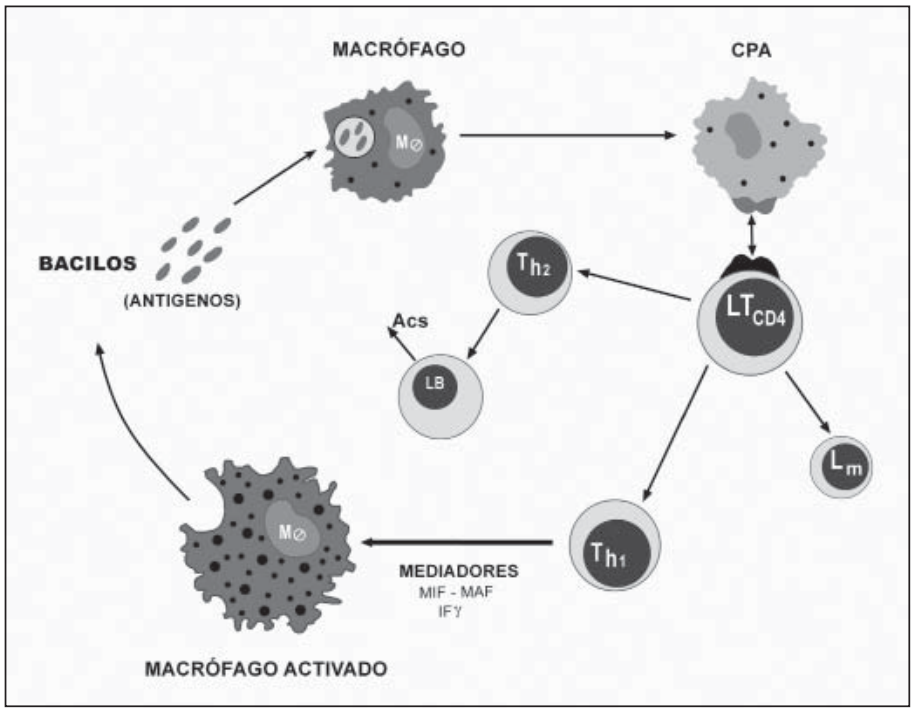

Figura 2. Respuesta celular inmune al $M$. tuberculosis. Ver texto. CPA: célula presentadora de antígeno; Acs: anticuerpos; IF $\gamma$ : interferón gama; MIF: factor inhibitorio macrofágico; MAF: factor activador macrofágico; L: linfocito; MØ: macrófago. individuo PPD negativo. También sabemos que la isoniacida es capaz de prevenir el desarrollo de tuberculosis en los sujetos infectados. Los resultados de una investigación realizada por la Unión Internacional Contra la Tuberculosis publicada en 1982 en 28.000 pacientes con lesiones fibróticas pulmonares, reveló que la isoniacida era capaz de prevenir la tuberculosis en más del 90\% de los casos que la tomaron durante 12 meses $^{25}$.

A comienzos del siglo veinte en Filadelfia Opie ${ }^{15,16}$, a partir de pulmones de enfermos fallecidos por otra causa diferente de la tuberculosis, inyectó extractos de tejido pulmonar a cobayos en la región inguinal para verificar cuántos de ellos desarrollaban la enfermedad. Pudo demostrar que las lesiones calcificadas tienen pocos bacilos, ya que sólo un $25 \%$ de los cobayos inyectados con extractos de estas lesiones desarrollaban tuberculosis. Pero, el hallazgo más importante fue que muestras de pulmones histológicamente normales, sin nada que hiciera pensar que tenían o habían tenido tuberculosis, eran capaces de producir tuberculosis en los cobayos.

Recientemente Hernández Pando ${ }^{17}$, en pulmones de pacientes fallecidos por otras causas, con la reacción de la polimerasa en cadena (PCR) demostró la presencia de ADN micobacteriano en pulmones que eran histológicamente normales. Los bacilos parecen estar no sólo en los macrófagos sino que también han sido detectados en fibroblastos y en otras células como los adipocitos.

Por otra parte, existen comunicaciones que dan cuenta de enfermos que han desarrollado tuberculosis después de treinta años de haber sido infectados ${ }^{18}$. Un caso notable es el referido en la literatura médica de un padre que infectó a su hijo en la infancia, el cual enfermó de tuberculosis 33 años después que el padre, con la misma cepa, a pesar de haber vivido separados por más de 20 años. Al comparar la cepa del hijo con más de 200 cepas provenientes de otros enfermos, con ninguna otra hubo un fingerprinting similar. En Dinamarca también pudieron comprobar patrones de ADN idénticos, al comparar cepas de los años sesenta con cepas de los ' 90 , en pacientes que diferían en 30 años, lo que es altamente sugerente de que la infección la adquirieron en los años ' $60^{19}$.

Todos estos argumentos han llevado a concluir que existen bacilos de Koch vivos, en los individuos portadores tuberculosis latente, aunque sin saber bien en qué forma.

\section{¿Cómo logra permanecer el bacilo vivo en forma latente en los tejidos?}

Existen por lo menos tres mecanismos que el bacilo puede desarrollar para permanecer latente sin ser eliminado del huésped y que le permite vivir en ambientes poco propicios ${ }^{20-22}$.

El primero es la capacidad del bacilo de interferir en la vía que procesa y presenta los antígenos mayores de histocompatibilidad de clase II del huésped; el segundo es la posibilidad de interferir con la fusión de los fagosomas y lisosomas y, por fin, su capacidad de interferir en la generación de óxido nítrico y productos intermediarios del oxígeno.

Desconocemos la forma fisiológica en que estos bacilos persisten en el organismo. ¿Qué grado de actividad metabólica mantienen?, ¿Permanecen inactivos como esporas? 


\section{¿Cuánto dura la infección latente?}

No contamos con un método diagnóstico de certeza, pero hay algunos estudios poblacionales que aportan alguna luz sobre este problema. Uno de los más convincentes es una investigación noruega publicada recientemente. En ella se siguieron cohortes por décadas de nacimiento desde 1876-1885 hasta 1959-1968, para evaluar los cambios ocurridos en la incidencia de tuberculosis en el país. Registraron los datos de los pacientes que desarrollaron tuberculosis desde 1946 hasta 1974, año en que terminaron el estudio para evitar la contaminación de infecciones exógenas derivadas de la inmigración ${ }^{23}$. Observaron que la tuberculosis fue disminuyendo en forma constante durante todos esos años, pero con una tendencia clara a una disminución más lenta a medida que se alejaba de la infección. Esto demostraría que el riesgo de reactivación disminuye con la edad, lo que refleja la frecuencia con que la infección latente es eliminada de la población, con menor tendencia a la progresión a enfermedad.

\section{¿En qué estado permanece el bacilo durante la infección latente?}

Habitualmente se piensa que un individuo infectado por el bacilo de Koch lo retiene en las lesiones antiguas durante el curso de su vida en estado "durmiente“. Así este bacilo sería el responsable de las reactivaciones futuras de la enfermedad con ayuda de factores de resucitación.

Sin embargo, hay al menos tres preguntas relevantes que deben ser contestadas $2,24,35$.

1. La isoniacida es una droga activa sólo para los bacilos que se están multiplicando y sabemos que es efectiva para prevenir el desarrollo de la enfermedad; por lo tanto, debería eliminar sólo los bacilos activos. Si éstos están realmente en estado "durmiente" su despertar podría ocurrir durante toda la vida, y así la quimioprofilaxis con isoniacida debería tomarse durante todo el tiempo en que ellos puedan despertar, es decir, de por vida. Sabemos que no es así, que bastan nueve meses para disminuir el riesgo de enfermar en forma importante.

2. ¿Cómo pueden permanecer los bacilos durmientes en el pulmón, considerando el turnover celular? Si los bacilos permanecen dentro de los macrófagos y la vida media de estos es de aproximadamente 3 meses, necesariamente cada 3 meses tendrían que cambiarse de "domicilio”.
3. ¿Quién provee el factor de resucitación para los bacilos durmientes? Se sabe que sólo los bacilos activos tienen factores de resucitación $^{38}$.

Todas estas interrogantes han llevado a plantear otras alternativas.

Hay quienes sugieren que el bacilo está permanentemente infectando nuevas áreas del pulmón, principalmente a través del fluido alveolar. En las infecciones crónicas como la tuberculosis, aparecen en la periferia de los granulomas unos macrófagos espumosos, que recibirían los bacilos que han salido al medio extracelular, pero como están activados, les impiden su multiplicación. Los bacilos que permanecen en estos macrófagos están adaptados a las condiciones adversas y viajarían dentro de las células en el fluido alveolar por vía endobronquial hacia otros sitios del pulmón. Si llegan, por ejemplo, a los vértices pulmonares donde las condiciones son más adecuadas para el crecimiento del bacilo, eventualmente pueden reactivarse y formar un nuevo foco, que si sobrepasa los mecanismos defensivos, produciría la enfermedad.

Este fenómeno puede estarse produciendo continuamente, pero va decayendo con el tiempo. Quizá esto explique que la reactivación es más probable en los primeros años después de la infección, ya que se sabe que el $80 \%$ de los infectados que enferman, lo hacen en los dos primeros años de la infección ${ }^{25}$, aunque más excepcionalmente puede aparecer mucho después.

Una observación interesante es que los inmigrantes de países con alta prevalencia de tuberculosis que se trasladan a países de baja prevalencia también desarrollan la enfermedad en los dos primeros años de su llegada al país huésped.

\section{¿A quiénes tratar?}

Hay evidencias claras que los pacientes VIH $(+)$, con PPD positivo, los contactos infantiles de pacientes tuberculosos, los portadores de lesiones residuales de origen tuberculoso, con PPD positivo no tratados anteriormente, y los convertores recientes del PPD, son los grupos que más se benefician con la quimioprofilaxis $2,31-34$.

En el último tiempo se han ido acumulando evidencias sobre el riesgo de hacer tuberculosis de las personas que reciben terapia anti FNT, las que cuando son PPD positivas o IGRA positivas también deberían recibir quimioprofilaxis ${ }^{30}$.

En cambio, esta indicación debe decidirse sobre bases individuales en otros infectados de ries- 
go de hacer tuberculosis, como son los pacientes con indicación de tratamientos inmunosupresores o corticoidales en altas dosis. En estos casos será importante considerar el tiempo y la magnitud de la inmunosupresión, la probabilidad de que tengan realmente una infección latente y el riesgo de toxicidad por la isoniacida o la droga a utilizar ${ }^{2}$.

Actualmente, a pesar de los avances en nuestros conocimientos, no tenemos una manera adecuada de saber qué infectados van a enfermar, y los tests disponibles, PPD e IGRAs, tienen muy baja capacidad para predecirlo. Por esto, frecuentemente la indicación de quimioprofilxis se basa en un adecuado juicio clínico.

\section{¿Cómo tratar a un portador de tuberculosis latente?}

Disponemos de dos esquemas de tratamiento de la infección tuberculosa (quimioprofilaxis) sobre los que existe buena evidencia de su eficacia. Por el momento debemos atenernos a las indicaciones del programa Nacional de Control de la Tuberculosis que recomienda el primero:

1) La isoniacida, administrada durante 9 meses, confiere una protección sobre el $90 \%$ en los sujetos que la toman regularmente. Pero, la quimioprofilaxis con isoniacida tiene el importante problema de la poca adherencia, que no supera el $60 \%$ de los que la inician. Por otra parte, su mayor inconveniente es la toxicidad hepática, que varía según las series entre 1 a $2 \%^{2,25,37}$.

2) La rifampicina, administrada diariamente durante cuatro meses es el esquema preferido en Estados Unidos y algunos países europeos como Inglaterra. Hay algunos trabajos que señalan que este esquema tiene una relación costo-efectividad superior a la administración de isoniacida durante nueve meses, además con mayor adherencia ${ }^{27}$.

La asociación de rifampicina-pirazinamida por dos meses, si bien ha probado ser muy efectiva en enfermos VIH $(+)$, tiene el problema de su hepatotoxicidad, por lo que no es un esquema recomendable.

Un estudio reciente demuestra que la asociación rifapentina-isoniacida una vez por semana, administradas en forma totalmente supervisada durante tres meses, no es inferior a isoniacida sola durante nueve meses y tiene mayor adherencia ${ }^{28}$.

Existen otros esquemas en estudio especialmente en pacientes VIH (+), como la asociación isoniacida-rifampicina diaria por tres meses o dos veces por semana bajo supervisión directa, con resultados similares a los obtenidos con isonia- cida 6-9 meses ${ }^{29}$.

A nivel de animales de experimentación, como modelos de tuberculosis latente, la asociación de moxifloxacino-rifapentina, una vez por semana, también ha demostrado ser tan efectiva como 9 meses de isoniacida sola.

\section{¿Cómo tratar al contacto de un enfermo con tuberculosis multirresistente?}

No hay estudios que avalen el tratamiento de los contactos de pacientes con tuberculosis multirresistente. Sólo existen recomendaciones basadas en la opinión de expertos ${ }^{39}$. El CDC (Center for Disease Control and Prevention) de EE.UU. recomienda la asociación de una quinolona con pirazinamida o de etambutol con pirazinamida durante 6-12 meses. Sin embargo, la eficacia de este esquema es desconocida y su tolerancia tampoco es óptima ${ }^{7}$.

\section{Bibliografía}

1-. WORLD HEALTH ORGANIZATION. Global Tuberculosis Control: a short update to 2009 reports. WHO/ HTM/TB/2009, 411 Geneva, Switzerland, WHO 2009.

2.- FARGA V, CAMINERO J A. Tuberculosis, Tercera Edición. Editorial Mediterráneo. 2011.

3.- HORSBURGH C R Jr, RUBIN E J. Latent Tuberculosis Infection in the United States N Engl J Med 2011; 364; 15: 1441-8.

4.- VON PIRQUET C. Frequency for tuberculosis in Falsepositive tuberculin skin test what is the absolute effect of BCG and nontuberculous mycobacterium. Int J Tuberc Lung Dis 101: 1192-204. Childhood JAMA 1907; 52: 675-8.

5.- FARHAT M, GREENAWAY C, PAIN M, MENZIES D. False positive-tuberculin skin test what is the absolute effect of BCG and nontuberculous mycobacterium. Int J Tuberc Lung Dis 101: 1192-204.

6.- MENZIES D. Interpretation of repeated tuberculin tests: boosting, conversion and reversion Am J Respir Crit Care Med 1999; 159: 15-21.

7.- $\quad$ CHANG P C, CHANG L Y, WU Y C, LU C Y, KAO H S, LEE C Y, et al. Age-specific cut-offs for tuberculin skin test to detect tuberculosis in BCG-vaccinated children. Int J Tuberc Lung Disease 2008; 12: 1401-6.

8.- RICHELDI L. Diagnosing Latent Tuberculosis Infection: guess who's coming to dinner? Am J Respir Care Med 2009; 180: 1-2.

9.- AHMAD S. New approaches in the diagnosis and treatment of latent tuberculosis infection. Respir Research 2010; 11: 169-85.

10.- ERKENS C G, KAMPORST M, ABUBAKAR I, BOTHAMLEY G H, CHEMTOB D, HAAS W, et al Tuberculosis contact investigation in low prevalence countries: an European consensus. Eur Respir J 2010; 36: 925- 49. 
11.- SOLOVIC I, SESTER M, GÓMEZ-REINO J J, RIEDES H L, EHLERS S, MILBURN H J, et al. The Risk of Tuberculosis related tumour necrosis factor antagonist therapies: a TBNET consensus statement. Eur Respir J 2010; 36: 1185-206.

12.- ANDERSEN P, DOHERTY T M, PAI M, WELDINGH $\mathrm{K}$. The prognosis of latent tuberculosis: can disease be predicted? Trend Mol Med 2007; 13: 175-82.

13.- DHEDA K, SCHWANDRE S, ZHU B, VAN ZYLSMIT RN, ZHANG Y. The immunology of tuberculosis: From bench to bedside. Respirology 2010; 15: 433-50.

14.- CHAN J, FLYNN J A. The immunological aspects of latency in tuberculosis Clinical Immunology 2004; 110: 2-12.

15.- OPIE E. Aronson J Tubercle bacilli in latent tuberculosis lesion and in lung tissue without tuberculosis lesions Arch Pathol 1927; 4: 1-21.

16.- OPIE E L. Tuberculosis for the lungs and bronchial lymph nodes. JAMA 1927; 89: 1293-6.

17.- HERNÁNDEZ-PANDO R, JEYANATHAN M, MENGISTU G, AGUILAR, OROZCO H, HARBOE M, et al Persistence of DNA from Mycobacterium tuberculosis in superficially normal lung tissue during latent infection. Lancet 2000; 356: 2133-8.

18.- ULLEBAEK T, DIRKEN A, BAESS I, STRUNGE B, THOMSENV O, ANDERSEN AB. Molecular evidence of endogenous reactivation of Mycobacterium tuberculosis after 33 years of latent infection. J Infect Dis 2002; 185: 401-3.

19.- LILLEBAEK T, DIRKSE A, VYNNYCKY E, BAESS I, THOMSENV $\varnothing$, ANDERSEN A B. Stability of DNA Patterns and Evidence of Mycobacterium Tuberculosis Reactivation Occurring Decades after the Initial Infection J. Infectious Dis 2003; 188: 1032-9.

20.- TUFARIELLO J M, CHAN J, FLYNN J L. Latent Tuberculosis: mechanisms of host and bacillus that contribute to persistent infection Lancet Infect Dis 2003; 3: 578-90.

21.- CHAN J, FLYNN J. The immunological aspects of latency in tuberculosis Clin Immunol 2004; 110: 2-12.

22.- KUSNER D J. Mechanisms of mycobacterial persistence in tuberculosis Clin Immunol 2005; 114: 239-47.

23.- WIKER H G MUSTAFA T, BJUNGE G A, HARBOE $M$. Evidence for waning of latency in a cohort study of tuberculosis. BMC Infect Dis 2010; 10: 37-46.

24.- CARDONA P J. A Dynamic infection hypotesis of latent tuberculosis infection 2009; 37: 80-6.

25.- International Union Against Tuberculosis. Efficacy of various durations of Isoniazid preventive therapy for Tuberculosis: five years of follow-up in the IUAT trial. International against Tuberculosis Committee on Prophylaxis. Bull World Health Organ 1982; 60: 555-64.

26.- DIEL R, LODDENKEMPER R, NIEMANN S, MEYWALD-WALTER K, NIENHAUS A. Negative and positive Predictive Value of a Whole Blood Interferon \{gamma\} Release assay for developing Active Tuberculosis. Am J Respir Crit Care Med 2011; 183: 88-95.

27.- HOLLAND D P, SANDERS G D, HAMILTON C D, STOUT J E. Costs and costs effectiveness of four Treatment Regimens for Latent tuberculosis Infection. Am J Respir Crit Care Med 2009; 179: 1055-60.

28.- STERLING T R. Three months of Rifapentin and Isoniazid for latent tuberculosis infection. N Engl J Med 2011; 365: 2155-66.

29.- MARTINSON N A, BARNES L G, MOULTON L H, MSANDIWA R, HAUSLER H, RAM M, et al. New regimens to prevet tuberculosis in adults with infection N Engl J Med 2011; 365: 11-20.

30.- SOLOVIC I, SEASTER M, GÓMEZ-REINO J J, RIEDER H L, EHLERS S, MILBURN H J, et al The risk of tuberculosis related to tumour necrosis factor antagonist therapies a TBNET consensus statement Eur Respir J 2010; 36: 1185-206

31.- MACK U, MIGLIORI G B, SESTER M, RIEDER H L, EHLERS S, GOLETTI D, et al. LTBI: latent tuberculosis infection or lasting immune responses to M Tuberculosis? A TBNET consensus statement Eur Respir 2009; 33: 956-73.

32.- BLUMBERG H M, LEONARD M K Jr, JASMER R M. Update on the treatment for Tuberculosis latent infection JAMA 2005; 293: 2776-84.

33.- BLASI F, CONSENTINI R, MIGLIORI G B, BOERSMA W. Steps forward in Tri and Tuberculosis update from the ERS Respiratory Infections Assembly. Eur Respir J 2009; 33: 1448-53.

34.- LANGE C, RIEDER H. Intention to test is intention to treat Am J Respir Crit Care Med 2011; 183: 3-4.

35.- CARDONA PJ, RUIZ-MANZANO J. On the nature of mycobacterium tuberculosis-latent bacilli. Eur Respir J 2004; 24: 1044-51.

36.- RUSSELL D. Who puts the tubercle in tuberculosis? Microbiology 2007; 5: 39-47.

37.- LALVANI A, PAREEK M. A 100 year update on diagnosis of tuberculosis infection. Br Med Bull 2010; 93: 69-84.

38.- SHLEEVA M O, BAGRAMYAN K, TELKOW M V, MUKAMOLOVA G V, YOUNG M, KEEL D B, et al Formation and resucitation of "non-culturable" cells of Rhodococus rhodochrous and Mycobacterium tuberculosis in prolonged stationary phase. Microbiology 2002; 148 (pt5): 1581-91.

39.- ATS. Targeted tuberculin testing and treatment of latent tuberculosis infection. This official statement of the American Thoracic Society was adopted by the ATS Board of Directors, July 1999. Joint Statement of the American Thoracic Society (ATS) and the Centers for Disease Control and Prevention (CDC). This statement was endorsed by the Council of the Infectious Diseases Society of America. (IDSA), September 1999, and the sections of this statement. Am J Crit Care Med 2000; 161 (suppl 4 pt2): S221-47.

Correspondencia a:

Dr. Juan C. Rodríguez D.

Servicio Médico-Quirúrgico Respiratorio

Instituto Nacional del Tórax

J.M. Infante 717

Santiago, Chile.

E-mail: jotarodriguez@123.cl 